\title{
THE
}

\section{Successfully Collecting Quantitative Data From Random Samples of Nursing Homes and Residents}

Skye N. Leedahl

University of Rhode Island, skyeleedahl@uri.edu

Follow this and additional works at: https://digitalcommons.uri.edu/hdf_facpubs

The University of Rhode Island Faculty have made this article openly available.

Please let us know how Open Access to this research benefits you.

This is a pre-publication author manuscript of the final, published article.

Terms of Use

This article is made available under the terms and conditions applicable towards Open Access Policy Articles, as set forth in our Terms of Use.

\section{Citation/Publisher Attribution}

Leedahl, S. (2017). Successfully collecting quantitative data from random samples of nursing homes and residents. SAGE Research Methods Cases. doi:10.4135/9781473992948

Available at: https://dx.doi.org/10.4135/9781473992948

This Article is brought to you for free and open access by the Human Development and Family Science at DigitalCommons@URI. It has been accepted for inclusion in Human Development and Family Science Faculty Publications by an authorized administrator of DigitalCommons@URI. For more information, please contact digitalcommons-group@uri.edu. 


\title{
Successfully Collecting Quantitative Data
}

\section{From Random Samples of Nursing Homes}

\section{and Residents}

\author{
Skye N. Leedahl \\ University of Rhode Island, USA \\ Discipline \\ Health [D4] \\ Sub-discipline
}

Health Services Research [SD-Hlth-8]

\section{Academic Level}

Postgraduate

\section{Contributor Biography}

Skye N. Leedahl, PhD, is an assistant professor at the University of Rhode Island (URI) in the Departments of Human Development and Family Studies, and Political Science and was hired as part of a Cluster Hire Initiative in Aging and Health. She teaches courses on research methods, 
program evaluation, aging and health policy issues, and environmental gerontology. She received a PhD in Social Work from the University of Kansas in 2013.

\section{Published Articles}

Leedahl, S. N., Chapin, R. K., \& Little, T. D. (2015). Multilevel examination of facility characteristics, social integration, and health for older adults living in nursing homes. Journals of Gerontology, Series B: Social Sciences, 70B, 111-122.

doi:10.1093/geronb/gbu112. Retrieved from

http://psychsocgerontology.oxfordjournals.org/content/70/1/111.short

\section{Abstract}

This case presents a rigorous approach used to successfully recruit and collect quantitative data from nursing home staff members and residents. The study presented in this case was conducted for my dissertation research. The goal of the study was to understand the complex phenomena of social integration for nursing home residents and its relation to health and well-being outcomes. To gather data, this study utilized a two-stage multilevel sampling technique to obtain a stratified random sample of nursing homes $(N=30)$ and a random sample of older adult residents from each of the nursing homes ( $N=140$, from each facility $n=3-6)$. This study utilized structured interviews with nursing home residents using a planned missing data design as well as brief surveys with staff members to collect data. Within the complex policy and program structures of nursing homes, the use of random sampling (as opposed to convenience sampling) at multiple levels (nursing home and resident) required the use of multiple, carefully planned steps. This included making phone calls, utilizing specific inclusion criteria, obtaining informed consent at multiple levels, and providing small monetary incentives. This case presents methodological considerations related to sampling, recruitment, and data collection. It also presents some of the 
lessons learned in collecting data for this study. This approach can be translated within various fields to recruit and gather health and related information from specialized populations.

\section{Learning Outcomes}

By the end of this case, students should be able to:

- Utilize ideas in this case to help design a study using random sampling at two levels for gathering quantitative data

- Explain how to utilize proper informed consent procedures for specialized populations (such as those living in institutional settings)

- Identify specific data collection decisions and strategies for maximizing response rates and meeting sample size goals

- Describe the use of structured interviews to collect survey data and the use of a planned missing data design

\section{Case Study}

\section{Project Overview}

Many health service researchers and social scientists conduct survey research to ask individuals questions about multiple social phenomena, past experiences, and attitudes. In doing so, they often gather cross-sectional, quantitative data. Although experimental and/or longitudinal designs are considered the most rigorous types of quantitative methodologies, there are design techniques that researchers can use to overcome some of the limitations to cross-sectional designs, particularly when wanting to gather information from samples that can be generalized to the greater population. For example, it is suggested that obtaining large representative samples, 
using standardized measures and collection procedures, and examining response rates are particularly important to designing rigorous cross-sectional studies (Kelley, Clark, Brown, \& Sitzia, 2003).

However, this can be particularly challenging when studying specialized populations, such as adults living in institutions due to additional requirements needed to obtain adequate informed consent. Special populations are "research participants who, because of age, incarceration, potential coercion, or less than full physical, mental, emotional, or other capabilities, may lack complete freedom or awareness to grant voluntary consent to participate in a study" (Neuman, 2011, p. 151). When studying special populations, it is important to take extra precautions to ensure potential participants are not coerced into taking part in the study.

Across the globe, many adults, due to physical, mental, or cognitive impairments, live in facilities that provide daily care and supports. It is essential to understand the experiences of individuals who live in facility settings, such as nursing homes, in order to guide service providers, promote high-quality services that are responsive and appropriate to the diverse needs of individuals, and inform policies that influence programmatic priorities and decisions of these facilities. In the United States, nursing homes are facilities that provide 24-hour long-term care services to those with chronic disease and/or disability who are unable to live at home (National Institute on Aging, 2015). However, in order to gather quantitative data from individuals living in nursing homes (as opposed to analyzing secondary data), careful consideration must be made due to the complex policy and program structures of these facilities, which are often at least partially government-funded. 
In this case, I present information on the methods used for my dissertation study that was carried out between March 2011 and March 2013. In this study, I was working to examine relationships between facility characteristics, multiple aspects of social integration, and health outcomes for older adults living in nursing homes. In order to do this, I utilized in-person interviews using a structured questionnaire with residents living in nursing homes, and nursing home administrators and social service directors completed brief survey questionnaires. The approach involved collecting cross-sectional data and analyzing the data using multilevel structural equation modeling. Therefore, individual-level (i.e., resident) and group-level (i.e., nursing home) data were used to answer research questions. This article focuses on presenting information about the study's recruitment and sampling strategy and the data collection procedures. It also includes a discussion of some of the lessons learned when conducting this study.

\section{Recruitment and Sampling Strategy}

The Human Subjects Committee of Lawrence (HSCL), the University of Kansas Institutional Review Board (IRB), reviewed and approved all recruitment and sampling procedures for this study. Furthermore, informed consent was obtained from every nursing home administrator, social service director, and resident. Because the residents are considered institutionalized, extra precautions were taken to ensure residents personally consented to participation and understood interview procedures prior to interviewers entering the nursing home. Three trained interviewers, including myself, made phone calls to administrators, followed up with social service directors, and completed interviews with residents. Overall, I utilized a two-stage multilevel sampling technique to obtain (a) a stratified random sample of nursing homes $(N=30)$ and (b) a random 
sample of older adult residents from each of the nursing homes $(N=140$, from each facility $n=3-6)$. The next two sections describe how I obtained the sample of nursing homes followed by how the nursing home residents in the study were identified.

\section{Nursing Home Sample}

The goal was to identify 30 nursing homes to participate in the study. This was because a sample size of at least 30 Level 2 units was needed to conduct multilevel structural equation modeling (Bickel, 2007; Kreft \& de Leeuw, 1998). In this study, Level 1 units were the nursing home residents (discussed in further detail below). In order to obtain a stratified, random sample of 30 nursing homes for this study, I compiled a list of nursing homes (i.e., nursing facilities licensed to provide skilled nursing care) within 15 counties in Northeast Kansas using a publicly available list of nursing homes. At the time of data collection, there were 78 nursing homes qualified for participation in the study from these counties. These counties, representing the most populated in the state, were chosen to limit the sample to nursing homes in areas with access to similar community resources and to ensure feasibility of data collection. As most of these counties are close to large cities, most of the facilities were larger $(M=100.64$ beds, standard deviation $[S D]=53.81$, range $=17-269)$ than the Kansas average $(M=81.78$ beds, $S D=43.10$, range $=17-298)$

I stratified the list of nursing homes into two groups: (a) facilities with more than 120 licensed beds (i.e., larger facilities) $(n=20)$ and (b) facilities with 120 licensed beds or less (i.e., smaller facilities) $(n=58)$. This was done in order to ensure adequate representation of nursing homes required to have a degreed social worker on staff (i.e., larger facilities) versus nursing homes not required to have a degreed social worker (i.e., smaller facilities). Because one of my 
research questions included a variable on the role of social workers, it was important to stratify the sample using this method.

Through previous research, I had developed relationships with nursing home associations and some nursing homes across the state, which aided in recruitment for the study. Recruitment procedures were as follows. All 78 nursing home administrators were mailed a letter describing the study's purpose. With the letter, I also included letters of support for the study from the forprofit and not-for-profit nursing home associations if the nursing homes were members of these organizations.

Next, using statistical software, the nursing homes were randomly ordered using Microsoft Excel and placed on one of three interviewer's lists. Each interviewer's list contained larger facilities and smaller facilities. Each interviewer made phone calls to administrators starting at the top of the lists until 30 agreed to participate, with the goal of obtaining at least $20 \%-30 \%$ of the total sample from larger facilities. Administrators were contacted three times via phone or email before ceasing contact. If administrators showed interest in participating at some point, we continued to contact administrators via phone or email until they either agreed or disagreed to participation or until we reached at least three additional contacts.

Once administrators agreed to have their facility participate, they directed us to the social service director. As a next step, the social service director had to agree to participate in the study and to assist in obtaining the random sample of nursing home residents from their facility.

\section{Resident Sample}


A power analysis for computing minimum sample size for root mean square error of approximation (RMSEA) indicated that 56 subjects would provide over $80 \%$ power $($ power $=0.80)($ Preacher $\&$ Coffman, 2006). However, to ensure adequate sample size for conducting structural equation modeling using maximum likelihood (ML) estimation, a sample between 100 and 150 is recommended (Schumacker \& Lomax, 1996), and a sample size of 120 satisfies the demand of the ML estimator (Little, 2013). Therefore, 120 was the target sample size for older adult nursing home residents in this study.

To obtain a random sample of nursing home residents from each nursing home, I provided the social service director a list of four inclusion criteria for residents eligible to participate in the study. The inclusion criteria were that (a) residents had to be at least 65 years of age, (b) residents had lived in the nursing home for at least 4 months (i.e., beyond a short-term rehabilitation stay), (c) residents did not have a legal guardian, and (d) residents did not have moderate-to-severe cognitive impairment. To ensure consistent definitions of cognitive impairment across nursing homes, I utilized criteria that all residents would have similar scores on due to policy requirements. These inclusion criteria were utilized to ensure each resident was considered an older adult according to U.S. Medicaid guidelines, was considered a long-term resident of the facility, could personally consent to participation, and was cognitively able to answer the questions on the survey.

Based on the inclusion criteria, the social service director identified residents from the facility who were eligible to participate in the study and compiled a list. We utilized standardized criteria to decide how many residents to try to interview. If the nursing home had 59 beds or less, we aimed for three nursing home residents; 60-90 beds, we aimed for four nursing home residents; and 90 or more beds, we aimed for five nursing home residents. We asked the social 
service director to ask 1-2 more residents than needed to meet the target sample size in case someone declined or was unavailable when we came to complete the interviews. This strategy proved to be very helpful and actually enabled us to exceed our sample size goal. When the facility had more residents than needed on their lists of eligible residents, the social service director gave them identification (ID) numbers (\#s) to ensure anonymity and then contacted us to randomly select participants. Once the random list of residents was determined, he or she then contacted the residents to ask about participation and receive permission for interviewers to come meet with them. The social service directors were given a recruitment script for discussing the study with residents and a permission form for residents to sign agreeing to have interviewers come to the nursing home. We then worked with the social service director to identify a date to interview the residents who agreed to participate. On the selected date, an interviewer or interviewers went to the nursing home and worked with the social service director to meet with each resident.

\section{Response Rates}

The final sample for the study included 140 nursing home residents from 30 nursing homes. The overall nursing home consent rate for this study was 38.9\% (47.4\% of larger facilities and 36.2\% of smaller facilities). This response rate is similar to other studies that have included nursing home samples (Simons \& Jankowski, 2007). Of the nursing homes who declined participation, we never connected with approximately $25 \%$ of administrators via phone or email, about $8 \%$ lacked staff resources and time for participation, about $4 \%$ were experiencing major facility and staff changes (e.g., renovations or administration changes) that kept them from participating, another $4 \%$ did not have residents that met the study inclusion criteria (e.g., facilities specializing 
in dementia care, facilities only for short-term rehabilitation residents), and about 3\% stated that their corporate offices did not agree with participation. In five cases, administrators agreed to participate, but social service directors did not consent, thus the facility did not participate in the study.

Between three and six nursing home residents participated in the study from each nursing home. The overall consent rate for residents in the study was $75.7 \%$ (140/185). Of the 45 residents who did not participate in the study, about $62 \%$ declined; $33 \%$ were unable to take part in the interview due to sickness, health concerns, or other scheduled appointments; and about $4 \%$ started the interview but could not finish for health reasons. Anticipated recruitment rate for residents was $60 \%-70 \%$ based on a study with a similar sample (Mahan, 2005). Across the 30 nursing homes, the consent rate for residents ranged from 50\% to 100\%. Generally, a 50\% response rate of individuals is considered representative of the group (Verran, Gerber, \& Milton, 1995).

\section{Data Collection Procedures}

Overall, I was responsible for coordinating all data collection efforts, and I completed 53\% of the interviews. In addition, two research assistants (one masters-level social work student and one PhD-level social work student) were hired to assist with recruitment efforts and resident interviews due to the size of the data collection. Thus, there were three trained interviewers who completed the interviews with the nursing home residents. We collected data over a 9-month period (between May 2011 and January 2012), and the bulk of the data was gathered in July and August. 


\section{Resident Interviews}

The older adult nursing home residents in the study were asked questions from a standardized survey during in-person interviews. For this type of research, residents' self-report of their experiences is considered the "gold standard" (Kane \& Kane, 2003; Rubinstein, 2000). Because of this, we did not permit the use of a proxy in this study. The proposed model for the study was designed to examine the social worlds of older adult nursing home residents, and this required asking a large series of questions related to residents' social integration. As suggested by Carp (1989), 1 hour is the maximum amount of time an interview should take with older adults in order to avoid fatigue. Furthermore, respondents are less likely to answer all questions in lengthy surveys, which can lead to high rates of non-responses (Graham, Hofer, \& MacKinnon, 1996), and in fact, when participants have fewer questions to answer or fewer repeated measurements, they are less likely to be fatigued and thus more likely to offer high-quality data (Raghunathan \& Grizzle, 1995).

Due to these various considerations, a planned missing data design was utilized to ensure the interviews did not take longer than $1 \mathrm{hr}$. This specialized methodological technique allows researchers to utilize the full set of questionnaire items while reducing respondent burden (Enders, 2010). Furthermore, a planned missing data design "allows researchers to leverage limited resources to collect data for 33\% more survey questions than can be answered by any one respondent" (Graham, Taylor, Olchowski, \& Cumsille, 2006, p. 323). Dattalo (2010) argues that social work researchers are ethically obligated to construct the smallest representative samples possible. This is particularly germane to the frail, older adult population and to this study. As 
such, a planned missing data design provided a cost-effective, time-efficient method for obtaining a lot of information on a smaller sample (Dattalo, 2010).

The three-form planned missing data design was used (Graham et al., 2006). With this design, items are divided into four item sets (X, A, B, and C). Questions in the X set were asked of every participant. This included all questions from the main construct in the study, one key question within each construct (i.e., reference variable), and all demographic variables. Then, the other questions were randomly assigned to A, B, or C set (see Table 1), and the order of the items was varied across the different sets of items to control for order effects. In the end, every participant answered some items from every measure. The three-form design is flexible, and it is acceptable to have an unequal number of questionnaire items on each item set (Enders, 2010).

\section{Table 1.}

Caption: Three-form planned missing data design.

\begin{tabular}{|l|l|l|l|l|}
\hline Form & Set X & Set A & Set B & Set C \\
\hline 1 & All & $\begin{array}{l}1 / 3 \text { of } \\
\text { variables }\end{array}$ & $\begin{array}{l}1 / 3 \text { of } \\
\text { variables }\end{array}$ & None \\
\hline 2 & All & $\begin{array}{l}1 / 3 \text { of } \\
\text { variables }\end{array}$ & None & $1 / 3$ of \\
variables \\
\hline 3 & All & None & $1 / 3$ of & $1 / 3$ of \\
variables & variables \\
\hline
\end{tabular}


Each form had approximately the same number of questions (Form $1=86$, Form $2=84$, and Form 3 =86). Participants were randomly selected to receive a form, and the number of participants completing each form was roughly equivalent (Form $1=45$, Form $2=50$, and Form $3=45)$.

The drawback of the three-form design is that some correlations, because they are based on only one-third of the sample, are tested with lower power. However, as Graham (2009) states, "virtually all of the possible drawbacks are under the researcher's control and can generally be avoided" (p. 566). Although power is lost when conducting a planned missing data design due to the loss in number of observations, the loss of power is gained back and thus is nearly fully recoverable through the data imputation process (Graham et al., 2006). Basically, any concerns about statistical analyses when using a planned missing data design can be alleviated when imputing data using statistical software. Utilizing the planned missing data design enabled me to collect a large amount of information and test a complicated model. However, it did require me to be extremely diligent (i.e., needing to double-check everything multiple times) when making the forms, entering the data, and analyzing the data. Having multiple researchers involved is highly suggested when using this technique.

\section{Training and Pilot Testing}

To increase rater reliability, all interviewers took part in a training about the study and study procedures. This training included in-depth information about recruiting, ensuring informed consent for all involved, going through the interview protocol, and processing payments. Prior to pilot testing, interviewers also practiced the interview protocol and met to discuss the questions and any issues that arose. Then, at the pilot site, the three interviewers completed interviews with 
older adult nursing home residents using the structured questionnaire. We did this in order to ensure respondents were able to answer the questions without difficulty and to verify the length of the interviews. At the pilot site, 12 residents were approached by the social service director regarding study participation, and 10 nursing home residents agreed to take part in the interviews.

Following the pilot test, all interviewers met to discuss each question and the overall process. Overall, the interviews provided meaningful information for each construct, and responses to each question varied considerably. Furthermore, we received positive feedback from the residents about the interviews, stating they thought the interview gathered important information across key components of their social worlds. The duration of each interview was approximately 1 hour, ranging from about 45 to $75 \mathrm{~min}$. The timing of the interviews was determined to be on target, and none of the residents indicated fatigue with the length of the interviews. We made a number of minor revisions to the structured questionnaire following the pilot test, none of which changed the substance or meaning of any of the constructs or indicators. The changes included minor wording changes (e.g., "in-person" instead of "with you physically") and clarifications (i.e., added examples) for various questions to ensure interviewers answered questions from the respondents similarly. Because the changes were minor, I was able to include the 10 pilot participants in the overall study.

\section{Standardized Measures}

The survey forms for the nursing home residents and the nursing home staff members were developed using multiple standardized measures for this study. For the nursing home residents, interviewers asked participants questions from the survey and recorded responses. For Likert 
scale questions, we provided laminated cards with extra-large print font (i.e., 20 point Arial font) so that residents could easily choose a response. Administrators were asked to fill out a brief survey called the Kansas Culture Change Instrument (KCCI) Leader Version, which was developed and validated by the University of Kansas School of Nursing for the Kansas Department on Aging (Bott et al., 2009). In order to gather information about the role of social work in the nursing homes, I developed a brief survey for social service directors. Administrators and social service directors were given the choice of either filling the survey out and giving it back prior to us exiting the facility or they provided a stamped envelope so that they could mail back the completed instruments. This ensured that staff across nursing homes were filling out the same survey, but doing so at a time that was convenient for them. I was able to obtain a $100 \%$ response rate for the nursing home administrator and social service director surveys using these methods. Follow-up emails were sent to remind individuals as needed.

\section{Data Collection and Compensation}

As interviewers, we strived to give residents' control over the time and place of the interview. Thus, we worked with social service directors and residents to schedule the interview around other activities they had planned for the day and let residents decide where they wanted to complete the interview (e.g., his or her room, quiet room, and dining room). When meeting with each resident, we worked to build an atmosphere of equality by requesting permission to sit and speak to each resident. As many of the interviews were completed in residents' room, we brought along chairs that could easily be placed in front of residents wherever they were sitting (e.g., recliners, wheelchairs) such that each interview was conducted at eye level. This also ensured we did not sit on residents' beds. Interviewers began each interview by going through 
informed consent procedures that involved further describing the purpose of the study and informing the residents of their right to refuse or withdraw from participation in the study at any time. We were committed to taking ample time with residents in order to explain the purpose of the study and to answer questions before obtaining informed consent. To facilitate reading, large print font was used on the informed consent form in a font that was easy to read.

To compensate individuals for participation in the study, each nursing home administrator, social service director, and older adult resident was offered US $\$ 20$ cash for personal use. The interviewers obtained the necessary information for subject payment processing, and each participant received a receipt form. Importantly, the project received a waiver of the requirement that Social Security Numbers be collected for nursing home resident participants. Nearly all residents (94.3\%) and administrators and social service directors (88.3\%) accepted the payment; the remaining declined or lived in nursing homes that did not permit payments to staff or residents.

Following data collection, missing data were imputed using multiple imputation, and multilevel structural equation modeling was used to analyze the data gathered in the study. See Leedahl, Chapin, and Little (2015) for details on study results.

\section{Practical Lessons Learned}

\section{Related to Providing Incentives}

Providing incentives to the nursing home residents was very important to the study and increased the generalizability of the results, as individuals were included in the study who would not have 
otherwise participated. For example, one woman with very low income who was eligible for the study was not interested in participating until she heard about the US\$20 incentive. She, according to the social service director, was not normally a talkative, engaged person. However, including the perspective of those less socially involved (like the particular woman referenced) was very important to the study in order to ensure a range of responses on the various social and health measures. The incentive was also critical for conveying to the residents how important their contribution was and helping them to know how much we appreciated their time. For example, one woman nearly cried when receiving her US\$20 incentive. She communicated that she had not actually "earned" money for many years and really appreciated knowing that her time and effort were valued. The incentive often served as a way for residents to stay motivated enough to finish answering all the questions, and it allowed interviewers to end the interview on a positive note with each resident.

Initially, we were going to provide gift cards to all the participants. However, we had a difficult time identifying one type of gift card that would work for all participants. After consulting with a couple of social service directors, we decided to provide cash incentives in order to ensure that individuals could spend the money as they please. We worked with IRB and the university grants office to identify processes for obtaining the cash and recording how the money was recorded and provided to participants. The cash was stored in a locked box in my office, and only the amount of money needed to complete interviews at one nursing home was provided to interviewers. Overall, utilizing cash proved to be an efficient way to provide the incentives, and this enabled us to quickly provide participants with their incentive rather than having them fill something out and wait in the mail for a check. 
The monetary compensation did prove to be somewhat problematic for consistency purposes because some nursing homes had policies against accepting compensation. For example, in one nursing home, we did not provide individual incentives to participants, but we were able to tell them that money would be donated to the nursing home due to their participation. In another nursing home, we simply did not offer the incentive to any of the participants.

\section{Related to Having Multiple Interviewers}

A strength of the study was that there were multiple interviewers who conducted the resident interviews, and all interviewers received training, practiced the interviews prior to conducting the study, and discussed strategies for potential follow-up questions. Importantly, having three interviewers was critical to the success of the study. Having multiple interviewers enabled us to collect data from the larger nursing homes in a single day, which was both cost- and timeefficient. It was ideal to have two interviewers go to a nursing home on the day of the interviews to talk with the residents and not feel rushed during the interviews. It worked best to conduct the interviews in the morning until about lunchtime or after lunch until about 3:00 p.m. It was possible for one interviewer to complete five to six interviews in 1 day, but having two interviewers helped ensure residents were interviewed during ideal times and helped avoid interviewer fatigue.

One limitation of the study was that I did not assess for inter-rater reliability. Doing so would have required an additional level of pilot testing, and the funding and time limitations of this study precluded this additional step. Another potential limitation was that the validity threat of social desirability bias may be present for residents and administrators. The data collection for 
this study involved self-report from the residents, which is considered the gold standard.

However, this does provide limitations because research has shown that older adults tend to focus on positive information as a mechanism for regulating their emotional experience; this is referred to as "positivity bias" (Hooyman \& Kiyak, 2011). Therefore, when answering questions, it is possible that older adults reported higher levels of support, engagement, and health than actual reality.

\section{Related to Social Desirability Bias}

Because interviewers did not have previous relationships with the residents, it is possible that residents did not feel comfortable informing the interviewers, for example, of sad feelings they were experiencing or a lack of support they received from family members. Alternatively, this could be viewed as a strength of the study because some of the residents may have actually felt more comfortable talking about some of these issues with the interviewers because they knew the information was confidential and that interviewers would not be communicating with staff or family members. Future research is needed to understand these issues and if or how they influence study results.

Related to nursing home-level information gathered in the study, administrators may have had a tendency to report higher levels of culture change involvement than actual reality due to current recognition of culture change as a best practice. For future studies, I suggest verifying the information by doing a physical environment analysis or having two staff members fill out the same survey.

\section{Related to Recruitment and Sampling}


While recruiting nursing homes and residents to participate in the study, it was important that the interviewers had flexible schedules and were able to go to the nursing home quite soon after talking with the social service director about which residents could participate. The nursing home staff were busy, and I found that they sometimes would forget about the plan if the interviews were scheduled too far out in advance. Having said that, once in the nursing home, the staff members seemed grateful to have researchers there and were very accommodating and helpful, particularly for initially introducing interviewers to the residents. The staff members seemed to appreciate that the interviewers did not require the staff to monitor the interviews or provide extensive accommodations for researchers. For example, having something as simple as a foldup chair enabled us to conduct interviews even in the smallest of rooms, without requiring the staff to identify a room or chair for the interview.

Regarding sampling, the study was conducted in Kansas, so this does limit the generalizability for the resident and the nursing home samples. However, every attempt was made to ensure random sampling at both the nursing home-level and the resident-level, so this improves the ability to generalize from the results. For feasibility reasons, limiting the study to one area in Kansas made this study possible. Interviewers were able to drive back and forth and conduct interviews all in 1 day. Therefore, travel costs could be minimized, and the "work hours" for study interviewers were feasible as all interviewers had classes to take or teach and other responsibilities that would have made overnight travel not possible.

It can be said that the nursing home sample was generally representative of the population of nursing homes included in this study. However, there were two exceptions: chain membership and occupancy rate. Nursing homes that were part of chains and those with lower occupancy rates were less likely to participate. As a probable explanation for the differences, 
there are additional levels of command in nursing homes with chain affiliation, and in this study, when we spoke with administrators from these nursing homes, they often told us they needed to contact the national office to get permission to participate. Many of these situations resulted in never hearing from the administrator again.

As a possible explanation for the differences in occupancy rates, research has shown that there is higher staff turnover in facilities with lower occupancy (Harrington \& Swan, 2003). In this study, nursing homes had a difficult time considering participation when they were experiencing administrator or social service director staff turnover or when administrators or social service directors were new to their jobs. As an example, two nursing homes initially agreed to participate in the study; however, by the time interviewers called to follow-up, a new administrator or social service director had started who had no information that the previous staff person in their position had agreed to participation. Both of these situations led to nonparticipation, as the new staff members stated that they had not developed relationships with the residents or were not yet comfortable with their jobs such that they felt comfortable signing up for the study. Related to this, it did seem to be important that social service staff members had close, positive relationships with residents. This helped ensure residents were comfortable having interviewers talk with them, and it also helped when corresponding with other staff because they knew who had invited us into the nursing home.

Related to the older adult sample, we included older adults with relatively high cognitive functioning in this study. This level of cognitive functioning was needed in order to ensure older adults could answer the questions, but this does provide limitations because most nursing home residents have some cognitive impairment. It is suggested that future work should be completed to better understand relationships between social integration and health for older adults who have 
moderate-to-severe cognitive impairments. Finally, although the sample sizes were rather substantial considering the data collection efforts and time frame of the study, the reality is that for advanced statistical analysis, like multilevel structural equation modeling, there were limitations to what could be determined from the data, particularly for between-level testing. Having more residents per nursing home would have enabled me to conduct additional multilevel analyses, such as hierarchical linear modeling.

\section{Conclusion}

During data collection, older adults living in the nursing homes communicated their willingness to participate in the research project and their appreciation for being able to contribute to something that could lead to future good. From a research standpoint, the amount of effort and time that was required to obtain informed consent from 30 different nursing homes and complete in-person interviews using survey methodology with 140 individuals was challenging but worth it. Importantly, results from this research were accepted into one of the top journals in the field of gerontology (i.e., Journal of Gerontology: Social Sciences). This means that the information has the potential to reach a wide audience and hopefully lead to a broader understanding of the topic.

\section{Exercises and Discussion Questions}

1. What steps did I take to ensure informed consent from nursing home resident participants in the study? Why was this so important to the study? 
2. How did I recruit nursing homes to participate in the study? How did this lead to recruiting nursing home residents to participate in the study? What are some of the limitations of both samples?

3. What practical considerations (e.g., number of interviewers, number of follow-up phone calls, and incentive amount) did I make when collecting data from nursing home residents that enabled me to successfully collect data at multiple levels?

4. Think of a group of people you are interested in studying who are affiliated with a particular program or institution, for example, children in elementary schools. Using this case and consulting a research methods textbook, answer these questions:

a. How would you use convenience sampling to study this group at two levels (individual-level and group-level)?

b. How would you use purposive sampling to study this group at two levels (individual-level and group-level)?

c. How would you use random sampling to study this group at two levels (individual-level and group-level)?

d. What are the benefits of conducting random sampling?

5. What other methods might I have used to collect information about facility characteristics, social integration, and health for nursing home residents?

6. Describe the planned missing data design in your own words. What are the strengths and limitations of using a planned missing data design? If needed, refer to the article by Graham et al. (2006).

\section{Web Resources}


Information on nursing homes in the United States:

https://www.nia.nih.gov/health/publication/nursing-homes

Computing power and minimum sample size for RMSEA:

http://www.quantpsy.org/rmsea/rmsea.htm

\section{References}

Bickel, R. (2007). Multilevel analysis for applied research: It's just regression! New York, NY: Guilford Press.

Bott, M., Dunton, N., Gajewski, B., Lee, R., Boyle, D., Bonnel, W., . . Rachlin, R. (2009). Culture change and turnover in Kansas nursing homes, for the Kansas Department on Aging. Lawrence: University of Kansas School of Nursing.

Carp, F. M. (1989). Maximizing data quality in community studies of older people. In M. P. Lawton \& A. R. Herzog (Eds.), Special research methods for gerontology (pp. 93-122). Amityville, NY: Baywood Publishing.

Dattalo, P. (2010). Ethical dilemmas in sampling. Journal of Social Work Values and Ethics, 7(1). Retrieved from http://www.socialworker.com/jswve/

Enders, C. K. (2010). Applied missing data analysis. New York, NY: Guilford Press.

Graham, J. W. (2009). Missing data analysis: Making it work in the real world. Annual Review of Psychology, 60, 549-576. 
Graham, J. W., Hofer, S. M., \& MacKinnon, D. P. (1996). Maximizing the usefulness of data obtained with planned missing value patterns: An application of maximum likelihood procedures. Multivariate Behavioral Research, 31, 197-218.

Graham, J. W., Taylor, B. J., Olchowski, A. E., \& Cumsille, P. E. (2006). Planned missing data designs in psychological research. Psychological Methods, 11, 323-343.

Harrington, C., \& Swan, J. H. (2003). Nursing home staffing, turnover, and case mix. Medical Care Research and Review, 60, 366-392.

Hooyman, N. R., \& Kiyak, H. A. (2011). Social gerontology: A multidisciplinary perspective (9th ed.). Boston, MA: Allyn \& Bacon.

Kane, R. L., \& Kane, R. A. (2000). Assessing older persons. New York, NY: Oxford University Press.

Kelley, K., Clark, B., Brown, V., \& Sitzia, J. (2003). Good practice in the conduct and reporting of survey research. International Journal for Quality in Health Care, 15, 261-266.

Kreft, I. G. G., \& de Leeuw, J. D. (1998). Introducing multilevel modeling. Thousand Oaks, CA: SAGE.

Leedahl, S. N., Chapin, R. K., \& Little, T. D. (2015). Multilevel examination of facility characteristics, social integration, and health for older adults living in nursing homes. Journals of Gerontology, Series B: Social Sciences, 70B, 111-122.

Little, T. D. (2013). Longitudinal structural equation modeling. New York, NY: Guilford Press. 
Mahan, T. L. (2005). Perceived control in older adults living in long-term care facilities. Retrieved from Proquest (AAT 3169416).

National Institute on Aging. (2015). Nursing homes: Making the right choice. Retrieved from https://www.nia.nih.gov/health/publication/nursing-homes (accessed 4 January 2016).

Neuman, W. L. (2011). Social research methods: Qualitative and quantitative approaches. Boston, MA: Pearson.

Preacher, K. J., \& Coffman, D. L. (2006, May). Computing power and minimum sample size for RMSEA [Computer software]. Available from http://quantpsy.org/

Raghunathan, T. E., \& Grizzle, J. E. (1995). A split questionnaire survey design. Journal of the American Statistical Association, 90, 54-63.

Rubinstein, R. L. (2000). Resident satisfaction, quality of life, and "lived experience" as domains to be assessed in long-term care. In J. Cohen-Mansfield, F. K. Ejaz, \& P. Werner (Eds.), Satisfaction surveys in long term care (pp. 13-28). New York, NY: Springer.

Schumacker, R. E., \& Lomax, R. G. (1996). A beginner's guide to structural equation modeling. Mahwah, NJ: Lawrence Erlbaum.

Simons, K. V., \& Jankowski, T. B. (2011). Factors influencing nursing home social workers' intentions to quite employment. Administration in Social Work, 32, 5-21.

Verran, J. A., Gerber, R. M., \& Milton, D. A. (1995). Data aggregation: Criteria for psychometric evaluation. Research in Nursing \& Health, 18, 77-80. 